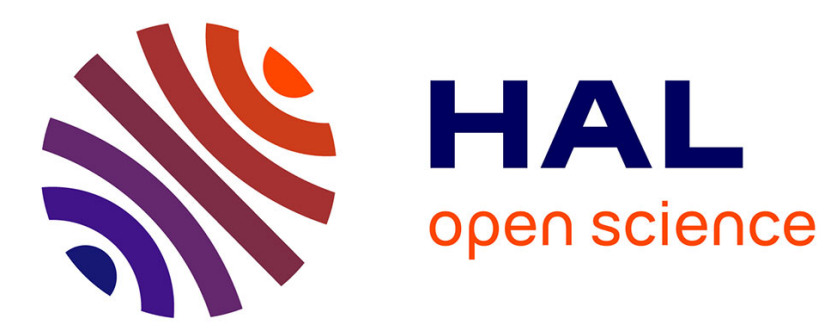

\title{
Civic Issues Reporting and Involvement of Volunteers as a Phenomenon in the Czech Republic
}

\author{
Miroslav Kubásek
}

\section{To cite this version:}

Miroslav Kubásek. Civic Issues Reporting and Involvement of Volunteers as a Phenomenon in the Czech Republic. 11th International Symposium on Environmental Software Systems (ISESS), Mar 2015, Melbourne, Australia. pp.151-159, 10.1007/978-3-319-15994-2_14 . hal-01328543

\section{HAL Id: hal-01328543 \\ https://hal.inria.fr/hal-01328543}

Submitted on 8 Jun 2016

HAL is a multi-disciplinary open access archive for the deposit and dissemination of scientific research documents, whether they are published or not. The documents may come from teaching and research institutions in France or abroad, or from public or private research centers.
L'archive ouverte pluridisciplinaire HAL, est destinée au dépôt et à la diffusion de documents scientifiques de niveau recherche, publiés ou non, émanant des établissements d'enseignement et de recherche français ou étrangers, des laboratoires publics ou privés. 


\title{
Civic Issues Reporting and Involvement of Volunteers as a Phenomenon in the Czech Republic
}

\author{
Miroslav Kubásek \\ Institute of Biostatistics and Analyses, Masaryk University, Brno, Czech Republic \\ kubasek@iba.muni.cz
}

\begin{abstract}
Today's smartphones can unlock the full potential of crowdsourcing and take eParticipation to a new level. Users are allowed to transparently contribute to complex and novel problem solving. Engagement of citizens is still challenging but the proliferation of smartphones with geolocation have made it easier than before. In this paper we will introduce the ZmapujTo project - a reporting platform in the form of a mobile application and responsive web page intended for citizens to report civic issues. We will also describe used technology stack and system architecture. In addition, we will introduce "Uklid'me Česko" (Clean up the Czech Republic) which is a maiden event taking place in the Czech Republic. We explain how ZmapujTo is applied in this event and the ICT tools that are offered to volunteers and organizers for communication and management of the event.
\end{abstract}

Keywords: Crowdsourcing · smartphones · illegal landfills · geolocation · web portal $\cdot$ GIS $\cdot$ mapping $\cdot$ human sensors

\section{$1 \quad$ Introduction}

Illegal waste dumping is a serious environmental concern in many countries[1,2]. These illegal dumps decrease the quality of human life in surrounding areas. Illegal dumping of garbage, discarded appliances, old barrels, used tyres, furniture, yard debris, oil, antifreeze and pesticides can threaten human health, wildlife and the environment.

Engaging citizens is challenging but due to the proliferation of personal smartphones with geolocation it is easier to develop a reporting system that can be used by every citizen. Timely elimination of illegal dump sites can limit the extent and severity of the damage (soil and water contamination, hazard of fire etc.).

Solving these issues was the main reason for creating the ZmapujTo. Project was started in mid 2011 as an e-Participation project and became the most popular environmental project in the Czech Republic. ZmapujTo is based on community phenomenon and thus leverages the power of crowd. It enables each one of us to be heard[3,4,5].

adfa, p. 1, 2011.

c) Springer-Verlag Berlin Heidelberg 2011 


\section{The ZmapujTo Web Portal}

In this chapter we shortly explain the framework of the Web portal ZmapujTo and introduce the technology of interactive mapping which enables detailed viewing of reported issues. We use several advanced GIS technologies to improve the visualization of reported illegal dumps (Fig. 1).

The first technology is the Google Street View ${ }^{1}$, which enables users to get a very detailed overview of the situation around the reported illegal dump. The Google Street View is a feature of Google Maps that provides $360^{\circ}$ panoramic street-level views and allows users to view parts of selected cities and their surrounding metropolitan areas at ground level. The Google Street View displays photos that were previously taken by a camera mounted on an automobile. Due to the fact that illegal dumps are mostly located near roads, we found the Google Street View as a useful tool to add a situation overview about the reported illegal dump.

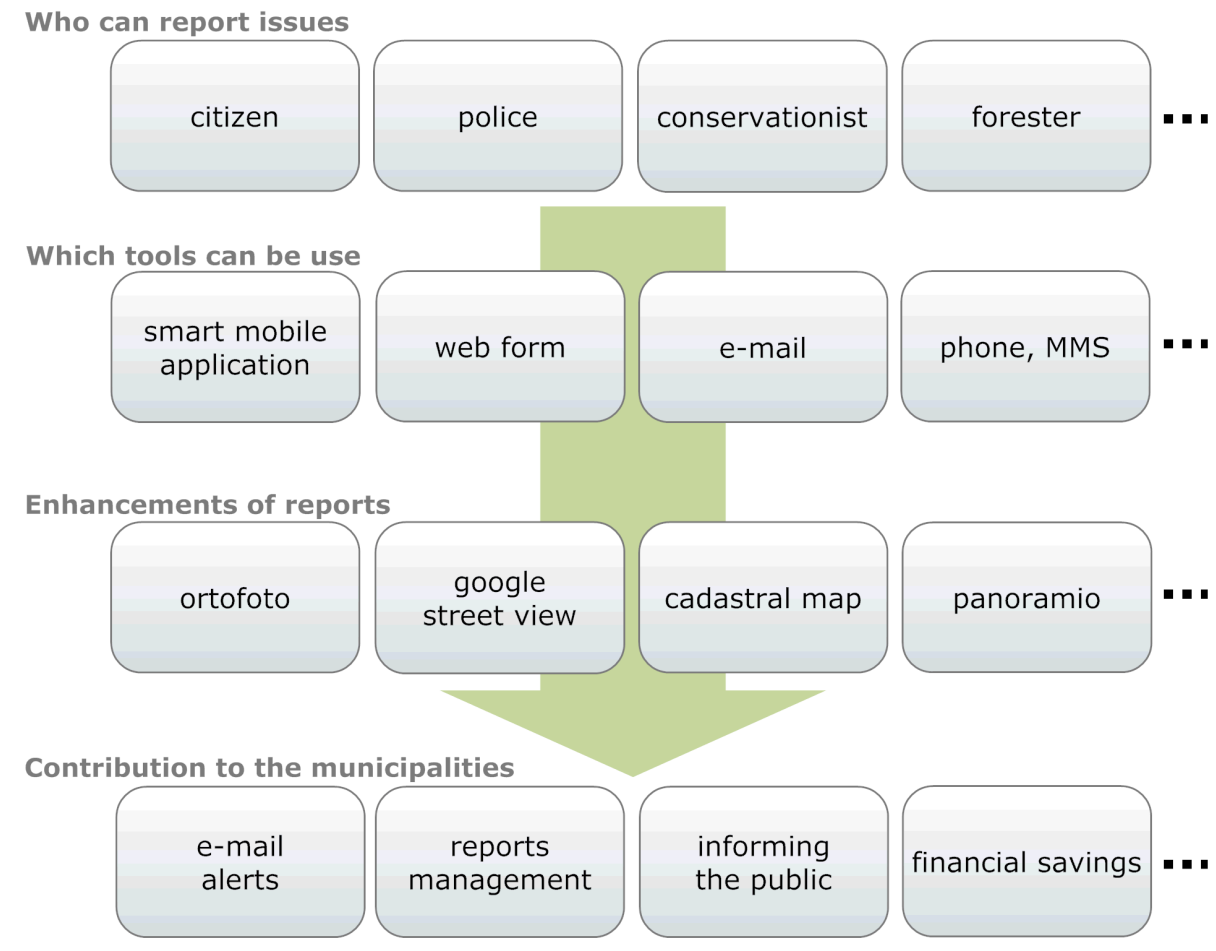

Fig. 1. Framework of the project ZmapujTo

1 http://maps.google.com/intl/cs_cz/help/maps/streetview/ 
Next we integrate the cadastral maps of the Czech Republic ${ }^{2}$ to the web portal ZmapujTo. This technology can simply identify the owner of the illegal dump site and project a specific plot of this. The municipal authority can thus directly contact this owner.

The map of illegal dump sites also provides a set of layers which can be explored by the users. The user can select from the menu of the portal the ortofoto layer, hybrid layer and a terrain layer.

We gained experience using the initial version of ZmapujTo portal since 2011 and we have collected a list of opinions, proposals and criticisms. For example a lot of municipalities wanted to extend the types of reports (we had only one possibility to report illegal dumps, so they proposed to extend reports e.g. about overloaded bins). Some authorities closely cooperated with their city police department to help monitoring illegal dumps in their cities. They were interested to integrate a reporting system directly into the police information and communication systems etc.

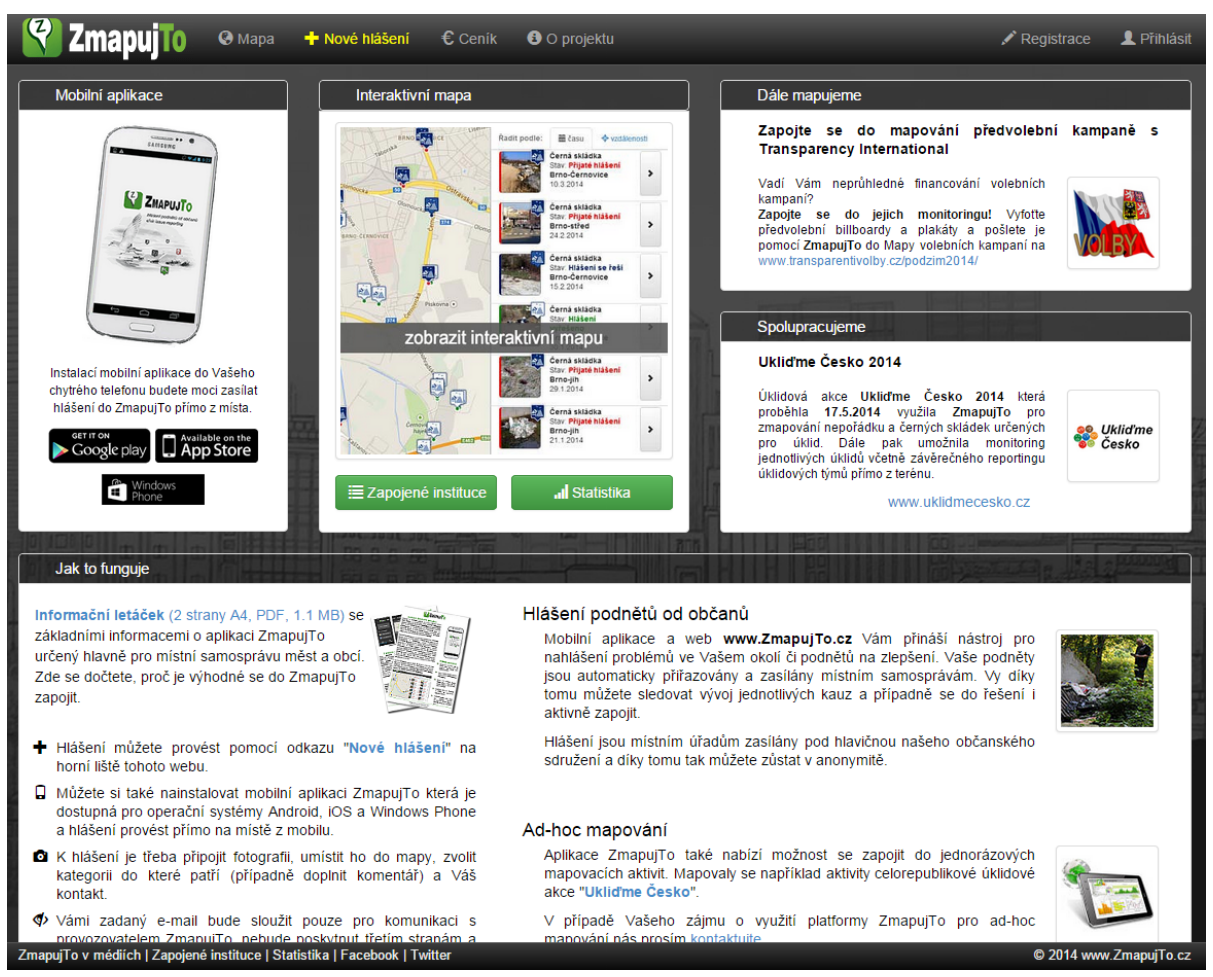

Fig. 2. Welcome page of ZmapujTo

Based on user feedback we launched a new version of the ZmapujTo portal in Spring of 2014 (Fig.2). The main properties of this new version are: an extension of report types, an extensive list of mobile platforms which will allow running the

2 http://nahlizenidokn.cuzk.cz/ 
ZmapujTo mobile application, and an improved web page to employ a responsive design paradigm and enable the use of tablets and phones to browse and manage reported issues.

\section{System Architecture}

The general system architecture (Fig. 3) is proposed to utilize existing technical solutions and services as much as possible. We have tried to design the system so that it can be deployed on a distributed environment if it will be necessary, e.g. in case of large traffic.

We analyzed the state-of-the-art information and communication technologies (ICT) and decided to use a single page application approach of SPA ${ }^{3}$ technology in order to improve the web portal response time. We selected the AngularJS ${ }^{4} \mathrm{JavaS}^{-}$ cript $^{5}$ framework. We also applied the JavaScript language in cooperation with NodeJS $^{6}$ (JavaScript event-driven runtime) and Express ${ }^{7}$ (the web application framework for nodeJS on the server side). We decided to leave traditional relation databases and used the document base $\mathrm{NoSQL}^{8}$ database MongoDB ${ }^{9}$ to store the data.

We selected the DigitalOcean ${ }^{10}$ platform for hosting (it offers SSD cloud servers from $\$ 5$ per month). Using this combination of ICT tools we have reached response times under $70 \mathrm{~ms}$ on user frontend events (e.g. map zoom, filter apply).

We also developed the new mobile application with Sencha Touch ${ }^{11}$ HTML5 framework and ported it to Android, iOS and Windows Phone devices through Apache Cordova ${ }^{12}$.

All work data of ZmapujTo are stored into the MongoDB database. This database contains collections of reports, users and also additional geographical data (ArcČR 500 version $3.1^{13}$ ). We widely used geospatial queries and we can say that in MongoDB they works without any problems. Data are backed up regularly (five times per day) on geographically remote location.

We do not store the images ourselves. Instead we use the excellent Cloudinary ${ }^{14}$ service for fast CDN-based image delivery. Due to limited storage capacity and bandwidth of Cloudinary we store here only processed images (resized to 500x500

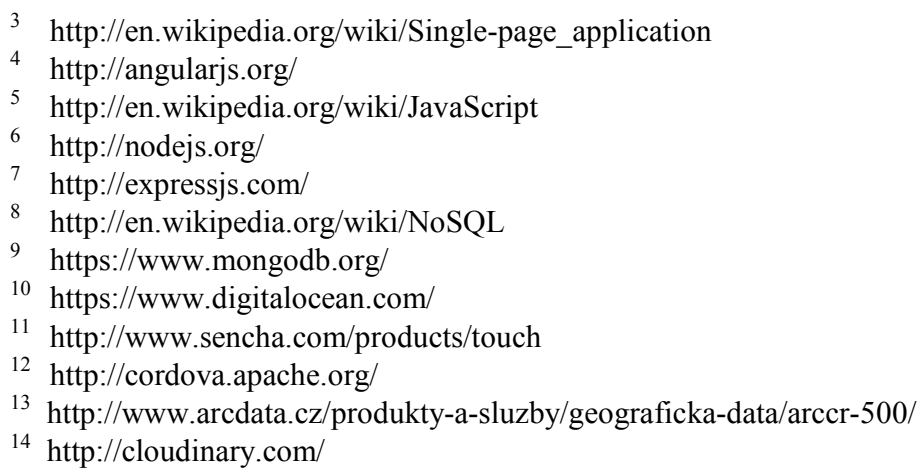


px). Original images we uploaded into Amazon S3 ${ }^{15}$ online file storage to keep them for possible future use.

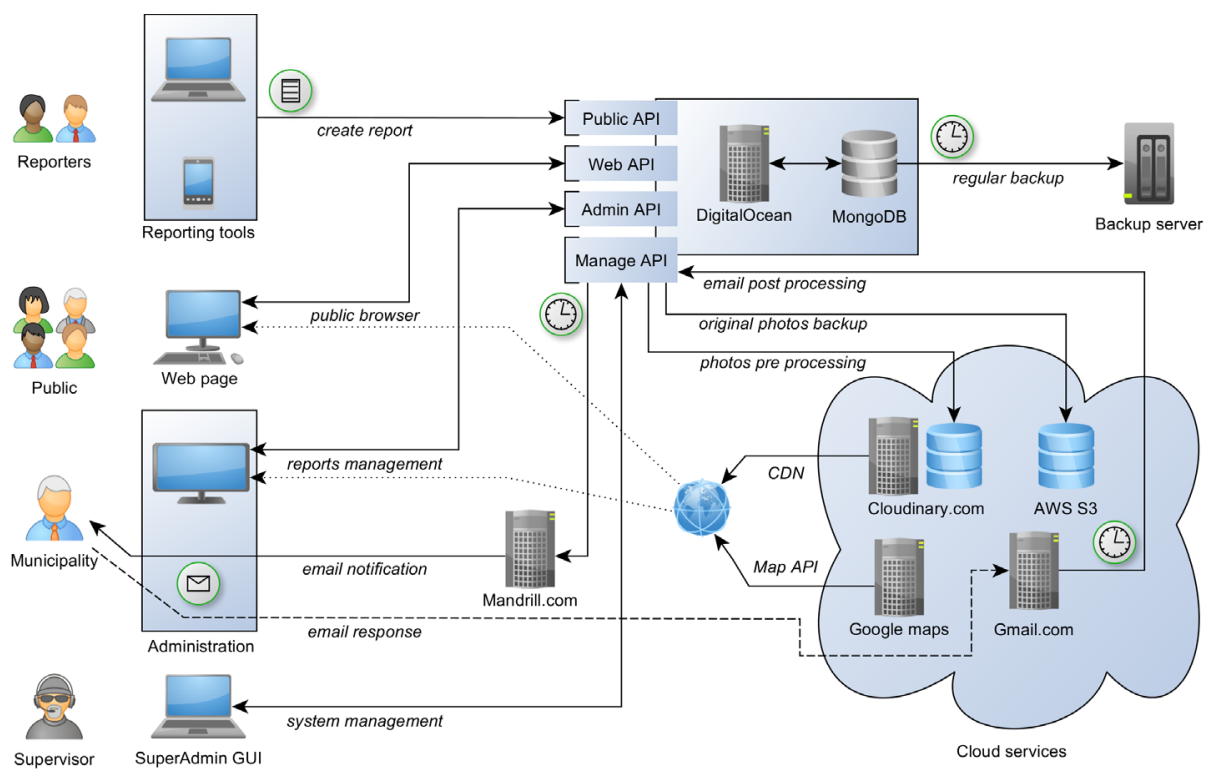

Fig. 3. System architecture

We created RESTful server with several APIs, where we combine the best of NodeJS and PHP worlds. We use NodeJS mostly for critical, the most widely used parts (e.g. filtering of reports in map, on-line providing of the data).

In our system we have proposed four APIs. The first "Public API" is intended to be a backend server for mobile applications. Its main objective is to receive photos and reports from mobile applications and from on-line form. The second API "Web API" is intended to serving data for web application (based on AngularJS framework). "Admin API" with authentication is intended to municipalities, which are able to manage reports in their regions. Last "Manage API" is used by supervisor and enable to manage and processing of new reports, processing of photos, management of email notifications etc.

For email notifications of municipalities we utilized the Mandrill ${ }^{16}$ which has a easy to use really good API. For processing of emails from municipalities we simply utilized Gmail ${ }^{17}$ service. We use email prefixing (append a plus "+" sign and any combination of words or numbers after your email address) to enable automatically unique identification of related report.

\footnotetext{
15 Amazon Simple Storage Service http://aws.amazon.com/s3/

$16 \mathrm{https} / / /$ mandrill.com/

17 https://mail.google.com
} 
The system in this configuration works very well for last 10 months. In this time period we did not notice loss of services used. System responses are despite the growing interest in ZmapujTo still very fast and sufficient.

\section{Efficient Report Management}

The key issue of the ZmapujTo reporting system is the communication with municipal authorities. In the ZmapujTo portal, the responsible municipality will receive information about the reported issue by e-mail. Municipal authorities have two possibilities to solve this event:

1. Either to answer directly to this email with response. Mostly it is information on whether they are aware of this issue or not. These responses are automatically processed and the content of these response e-mails are inserted directly to the record list of specific events;

2. Or to log-in into ZmapujTo web portal and process the report directly in its administration interface (Fig. 4). The aim of this application is to provide a simple tool, which will give to the authorities actual information about reported issues in their district. The administrator of the municipality assigned to a specific issue can change its, change location or leave comments as well. The administrator can also use the statistics of the district and a set of widgets (Fig. 5), which can be placed into the municipality web site and thus inform the visitors that the municipality is involved in the project, and how many issues have been reported and solved

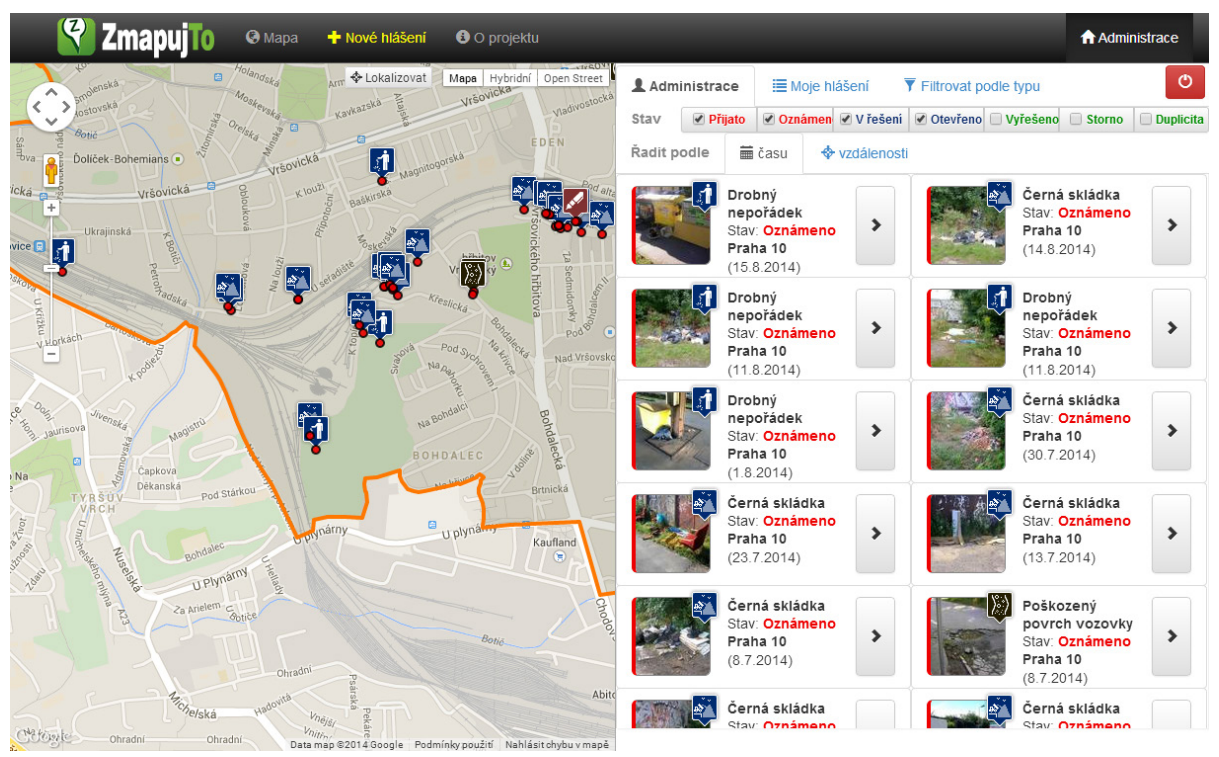

Fig. 4. Reports management and interactive map 


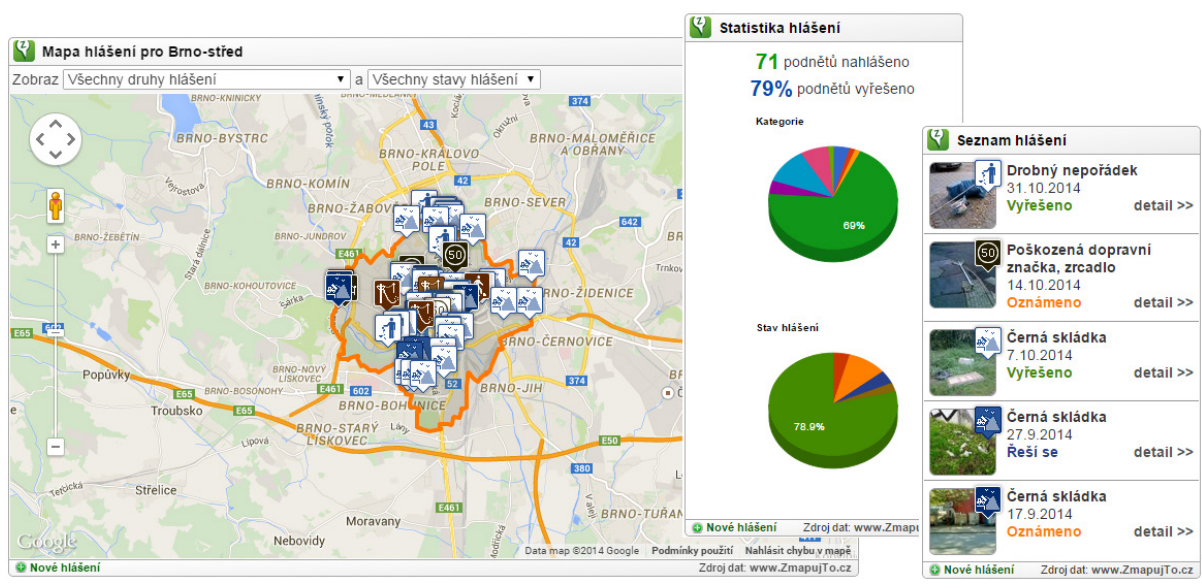

Fig. 5. Widgets which can by placed into municipality webpage

Currently, 470 municipalities and institutions from the entire Czech Republic are involved into ZmapujTo.

\section{Voluntary Event}

In 2014 for the first time, an event called "Uklid'me Česko" (Clean up the Czech Republic) took place in the Czech Republic (Fig. 6). It was inspired by past events from abroad (e.g. "Let's do it!", ${ }^{18}$ ), and hopes to address the continued lack of success in cleaning the dumps. The main goals were to warn and inform the public about the problems of illegal dumps and subsequently clean them up with the help of volunteers.

The cleaning was scheduled for 17th May 2014. This cleaning event collaborated closely with the ZmapujTo project and used its platform for mapping and registration of illegal dumps. The event was driven by volunteers, municipalities and nongovernmental organizations.

"Uklid'me Česko" participants were divided into groups, with having at least one organizer per group. The organizer was responsible for arranging group meetings in order to discuss various issues regarding the event.

One volunteer in the group used an interactive map (Fig. 7) on the web portal to register. Dark-colored points are drawn on the map to show all the registration points. Each of these points contains information about the number of registered participants (organizers and volunteers separately) in a particular location. Therefore one can check whether there is any organized group already present in his place of interest or

$18 \mathrm{http}: / / w w w . l e t s d o i t w o r l d . o r g /$ 
whether it is necessary to establish a new one. Those interested persons can register to one particular point at any part of a city or a village.

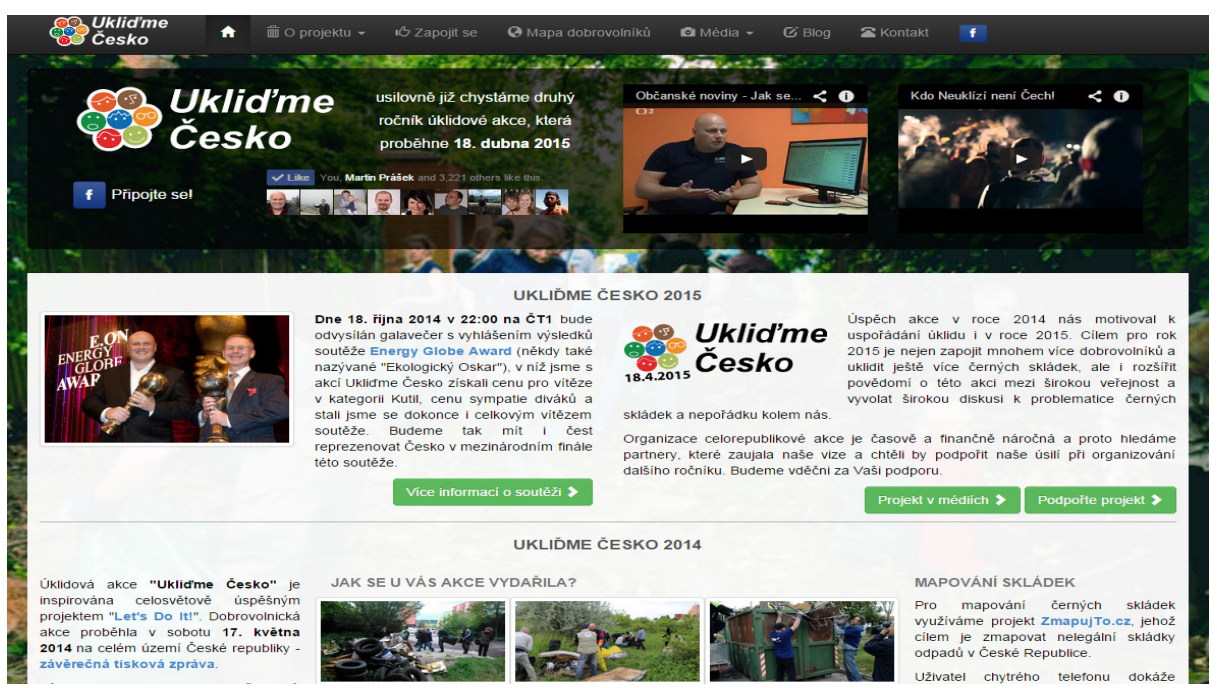

Fig. 6. Web page of the cleaning event

Thanks to the registration in this information system, organizers gain access (password required) to their account created directly on the web portal. Each organizer can manage a number of volunteers, choose a meeting point and an cleaning area (illegal dumps from ZmapujTo project are seamlessly integrated, so the organizer can select specific illegal dumps, which going to be cleaned up). This organizer's account also mediates communication with associated volunteers and with other organizers, for example from their neighborhoods.

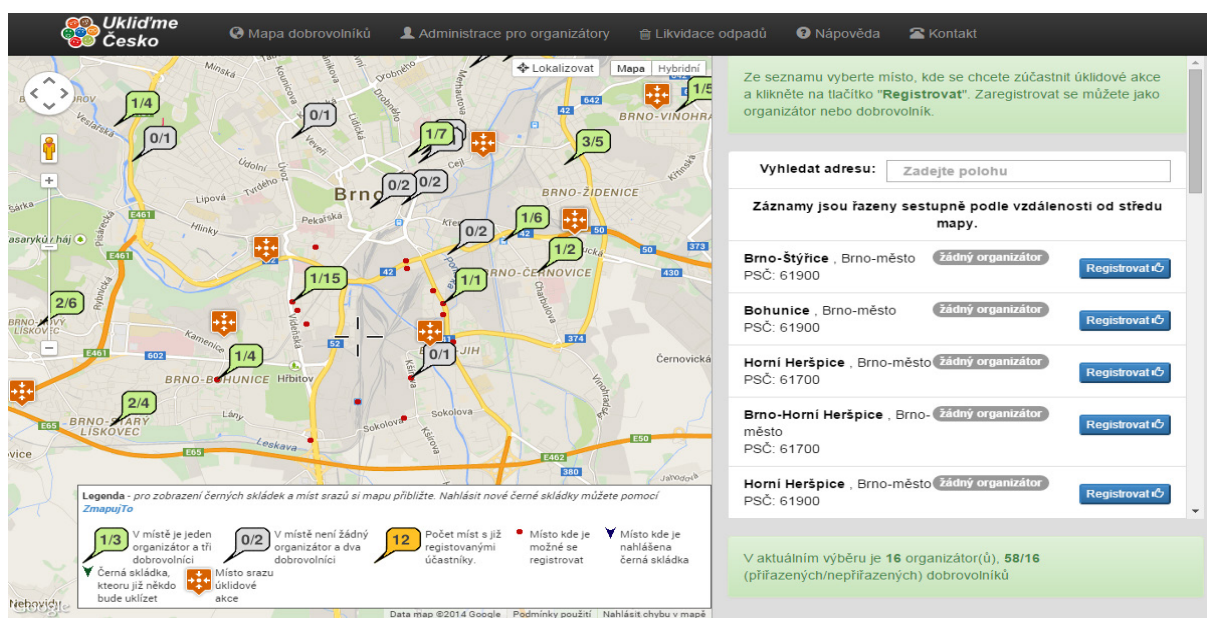

Fig. 7. Interactive map of volunteers integrated with illegal dumps from ZmapujTo 


\section{Conclusion}

The ZmapujTo project fulfills its aim to inform the public of the Czech Republic about the dangers related to illegal dump sites, motivates the public to report them, and offers municipalities and other organizations a tool for administration of these reports.

We followed various feedbacks collected from users and developed a new version of the web portal with state-of-the-art information technologies. An extension of the report types and automatic communication with all municipal authorities in the Czech Republic are the main benefits of the new version. The user-friendliness has been improved by using up-to-date and more efficient technologies. We utilize existing technical solutions and services as much as possible. We chose young technology as a NoSQL MongoDB database, Node.js, Express web framework and AngularJS for creating Single Page Application. Despite the initial fears, we can tell now, that during the operation of the system we didn't encountered a single failure or a major problem.

During the event "Clean up the Czech Republic" which took place on Saturday 17th May 2014 the participants collected 350 tons of garbage, the volunteers participated in 280 places over the whole country. Based on feedback from the organizers and volunteers who used the ZmapujTo mobile application and the interactive map of volunteers we can state that the our systems fulfills our expectations.

\section{References}

1. Europol, 2011. Europol warns of increase in illegal waste dumping. $<$ https://www.europol.europa.eu/content/simplenews/europol-warns-increase-illegalwaste-dumping-1057> (last accessed 15.03.14.).

2. HCCREMS, 2013.Hunter \& Central Coast Regional Environmental Management Strategy Illegal Dumping, $<$ http://www.hccrems.com.au/Programs/EnvironmentalCompliance/Sub-projects/Illegal-Dumping.aspx $>$ (last accessed 15.03.2014.).

3. Kubásek M., Hřebíček J., 2013. Crowdsource Approach for Mapping of Illegal Dumps in the Czech Republic. International Journal of Spatial Data Infrastructures Research, 8, 144157.

4. Kubásek M., 2013. Mapping of Illegal Dumps in the Czech Republic - Using a CrowdSourcing Approach. In: Hřebíček, J., Schimak, G., Kubásek, M., Rizzoli, A.E., (Eds.), Environmental Software Systems : Fostering Information Sharing. IFIP AICT vol. 413. Springer, Heidelberg, pp. 177-187.

5. Kubásek M. a Hřebíček J., 2014. Involving Citizens into Mapping of Illegal Landfills and other civic issues in the Czech Republic. In Ames, D.P., Quinn, N.W.T., Rizzoli, A.E.. Proceedings of the 7th International Congress on Environmental Modelling and Software. San Diego: iEMSS, 2014. ISBN 978-88-903574-4-2. 